\title{
Diffuse Alveolar Hemorrhage Associated with Dabigatran
}

\author{
Kensuke Yokoi, Kikuo Isoda, Toyokazu Kimura and Takeshi Adachi
}

Key words: dabigatoran, atrial fibrillation, diffuse alveolar hemorrhage, fresh frozen plasma, mechanical ventilation

(Intern Med 51: 2667-2668, 2012)

(DOI: 10.2169/internalmedicine.51.8345)
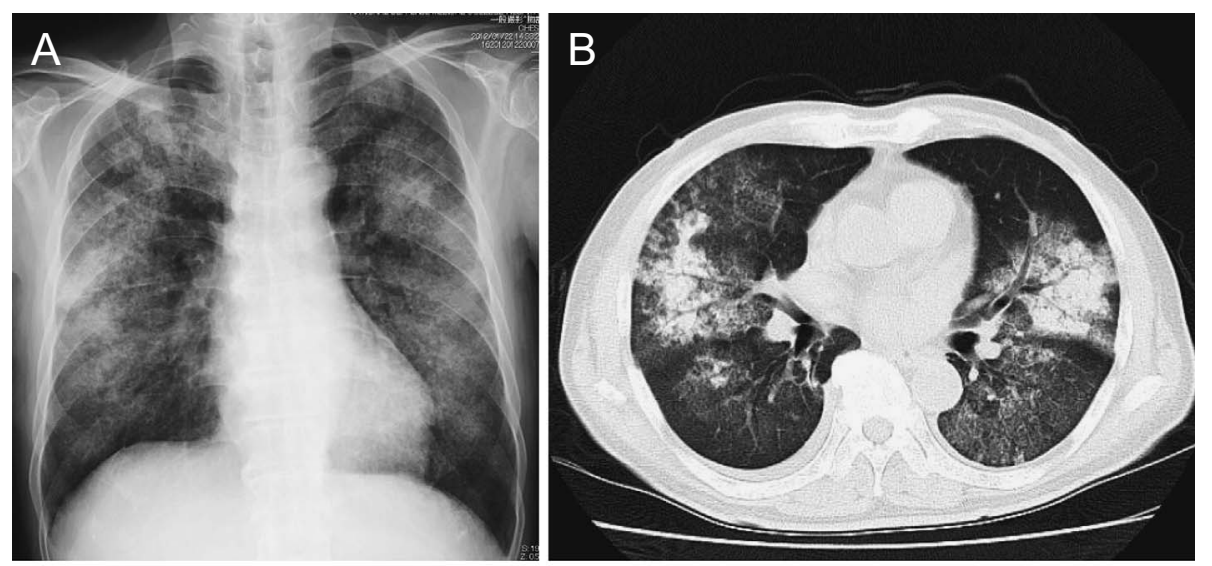

\section{On admission}

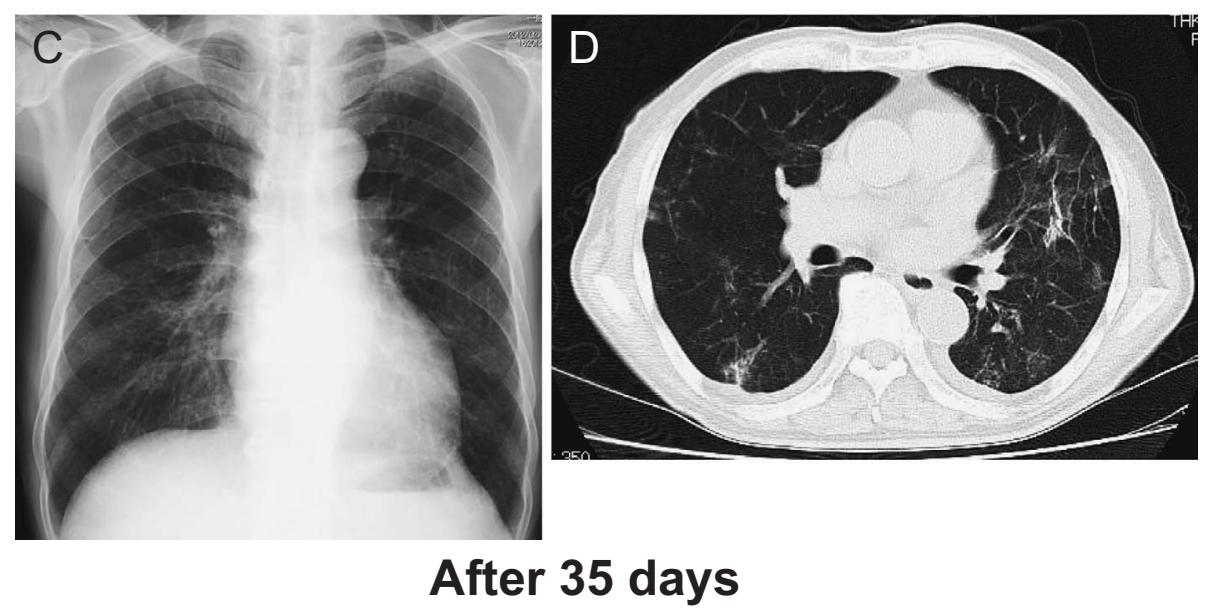

Picture.

A 78-year-old man with a history of paroxysmal atrial flutter and fibrillation that had been treated with dabigatran for two months was admitted with hemoptysis and dyspnea. Chest radiography and computed tomography (CT) showed bilateral alveolar infiltration (Picture A, B). His coagulation test results revealed a prothrombin time of $97.5 \mathrm{~s}$ and an international normalized ratio of 1.42. A diffuse alveolar hemorrhage (DAH) was clinically suspected and subsequently confirmed by the histological findings of hemosiderin-filled macrophages in his bloody bronchoalveolar fluid. The pa- 
tient required aggressive treatment with fresh frozen plasma and mechanical ventilation to achieve a successful outcome. Chest radiography and CT revealed an improvement of the bilateral alveolar infiltration after 35 days (Picture C, D). This is the first report of DAH caused by $220 \mathrm{mg}$ daily dabigatran therapy without any anticoagulation or antiplate- let agents. DAH associated with dabigatran, which has no effective reagent to reverse its activity, is a dangerous occurrence and early intervention must be carried out.

The authors state that they have no Conflict of Interest (COI).

(C) 2012 The Japanese Society of Internal Medicine http://www.naika.or.jp/imonline/index.html 